\title{
Research on the resilience of the intelligent integrated energy systems
}

\author{
E A Barakhtenko ${ }^{1}$, D V Sokolov ${ }^{1}$, A V Edelev ${ }^{1}$ and S A Gorsky ${ }^{2}$ \\ ${ }^{1}$ Melentiev Energy Systems Institute of Siberian Branch of SB RAS, Lermontov St. \\ 130, Irkutsk, Russia, 664033 \\ ${ }^{2}$ Matrosov Institute for System Dynamics and Control Theory of SB RAS, Lermontov \\ St. 134, Irkutsk, Russia, 664033 \\ flower@isem.sei.irk.ru
}

\begin{abstract}
The paper addresses the research of intelligent integrated energy systems resilience that is the system ability to withstand large disturbances and prevent their cascade development with massive energy shortage. The scheme of intelligent integrated energy systems resilience research is presented in the paper. It involves the disturbance scenarios formation, the evaluation of consequences of disturbance impact on energy systems, the assessment of the effectiveness and feasibility of the resilience enhancement steps. The scheme helps an expert to select specific resilience enhancement steps and to make decision whenever to implement them.
\end{abstract}

\section{Introduction}

The emergence of new information technologies and energy equipment, new requirements for energy systems, changes in the conditions in which they operate, contributed to the development of the concept of intelligent integrated energy systems (IIES) [1]. The IIES refers to systems that are focused on the use of several types of energy carriers with the use of information technologies and telecommunications. They together provide the creation of a more efficient system of energy production, energy supply and energy consumption.

In addition to reliability, IIES has the following distinctive properties aimed at providing high-quality and timely customer requests [1]:

- Flexibility - adaptation to any external disturbances and relevant current consumer needs.

- Intelligence - the ability of the system to timely and adequately respond to customer requests.

- Integration - supply systems integration, fitting into the territorial engineering infrastructure.

- Efficiency - compliance with energy efficiency requirements.

- Competitiveness - a combination of economic efficiency and availability of energy resources for consumers. Consumers have the ability to control their energy consumption to reduce the amount of payment for it.

- Net-centric - control of energy supply and energy consumption. The control is based on an extensive energy network, in which each element of the system is able to interact with any other element through a telecommunications network.

Thus, the IIES envisages the integration of traditional energy systems with new information and communication technologies and an integrated multi-level automated control system.

IIES have a multi-dimensional structure of functional characteristics and expansion properties. They combine a great number of components, intelligence, efficiency, reliability, controllability, flexible use 
of technologies for energy conversion, transportation storage, and active consumer [1]. Conceptually, the integration is carried out in three aspects [1]:

- A system aspect which represents the integration of systems by their type (electricity, heat/cooling and gas supply systems),

- A scale aspect which reflects the size of the systems with their differentiation into super-, miniand microsystems,

- A functional aspect which determines the functions of the system (its purpose), including energy (technological); communication, control and decision making.

In terms of the system aspect, the IIES is represented by the key infrastructural energy systems that can be highly integrated with respect to the functional tasks, mutual redundancy, technological interrelations at various hierarchical levels, etc.

In terms of the scale aspect, we distinguish the following interrelated systems [1]:

- Super-systems, i.e. traditional centralized energy supply systems that consist of large-scale electricity and heat sources, gas fields, underground gas storages, electrical, gas supply and heat supply networks,

- Mini-systems, i.e. decentralized (distributed) systems including mini electricity and heat sources (including those nonconventional and renewable), which are connected to the distribution electrical, thermal and gas networks, and these networks themselves,

- Micro-systems, i.e. individual systems with nonconventional and renewable electricity and heat sources as well as house electrical, heat and gas networks.

Functional aspects of IIES include the following constituent functions:

- Energy functions that represent production, transportation, distribution and consumption of electricity, heat/cooling, gas at all levels and scales,

- Functions of communication and control that represent measurement, processing, transfer, exchange and visualization of information, control of operating conditions and expansion of the metasystem,

- Decision-making functions, i.e. the metasystem intelligence which includes models and methods for planning the expansion of the integrated energy systems as well as settings for their control.

All the functional properties of the IIES have strong interrelations with one another in terms of input and output state variables, the structure of forecasts both at the level of operation and at the level of expansion. They form an absolutely new technological architecture which defines the organization of the metasystem implementing the design solutions of its components, their interactions with one another and with the external environment.

\section{Resilience conception}

The term resilience has increasingly been seen in the research literature over the last decade. The common use of resilience word implies the ability of an entity or system to return to normal condition after the occurrence of an event that disrupts its state [2]. Several definitions of resilience have been offered in $[3,4]$.

There are four domains of resilience: organizational, social, economic, engineering. The engineering domain includes technical infrastructures designed by engineers that interact with humans and technology, such as energy systems [2]. Due to the crucial role of such infrastructures on society and economy, they are often called critical and research work has recently focused on critical infrastructure resilience $[5,6]$.

In the context of critical infrastructures, resilience can be developed by focusing on the different stages of critical infrastructure performance change following a disruption event and developing strategies and improvements which strengthen critical infrastructure response.

\section{IIES resilience research process}

The resilience of IIES is the system ability to withstand large disturbances and prevent their cascade development with massive energy shortage [3]. 
Studies of the IIES resilience are associated with large disturbances which strain the system structure and behavior and lead to severe consequences for consumers. The conception of IIES resilience is associated with the magnitude of their consequences for IIES that characterizes the IIES adaptive capabilities to counteract and absorb large disturbances [4-7].

Thus the purpose of the IIES resilience research is to provide effective management of the IIES development and functioning to overcome the impact of large disturbances. The domain of IIES resilience research is the process of disturbances formation, the IIES reaction, the consequences for consumers, and the way to compensate for negative consequences [8-10].

The main blocks of IIES resilience research process [11] are presented in figure 1. IIES resilience research is started with collecting information about the IIES current state and development strategies, the disturbance types, the steps to improve IIES resilience. Information on the IIES current state and development strategies is the result of traditional general energy research. The considered types of disturbances and steps to improve resilience depend on the goals of resilience research.

The first block generates possible IIES states which reflect the most representative combinations of external conditions of their development and functioning in the considered time interval. Also, it generates disturbance scenarios.

The second block applies disturbance scenarios on the possible IIES states, evaluates consequences of disturbance impact on IIES and identifies the IIES critical elements. The disturbance is usually simulated as the IIES structure deformation and the reduction of the functional properties of IIES elements.

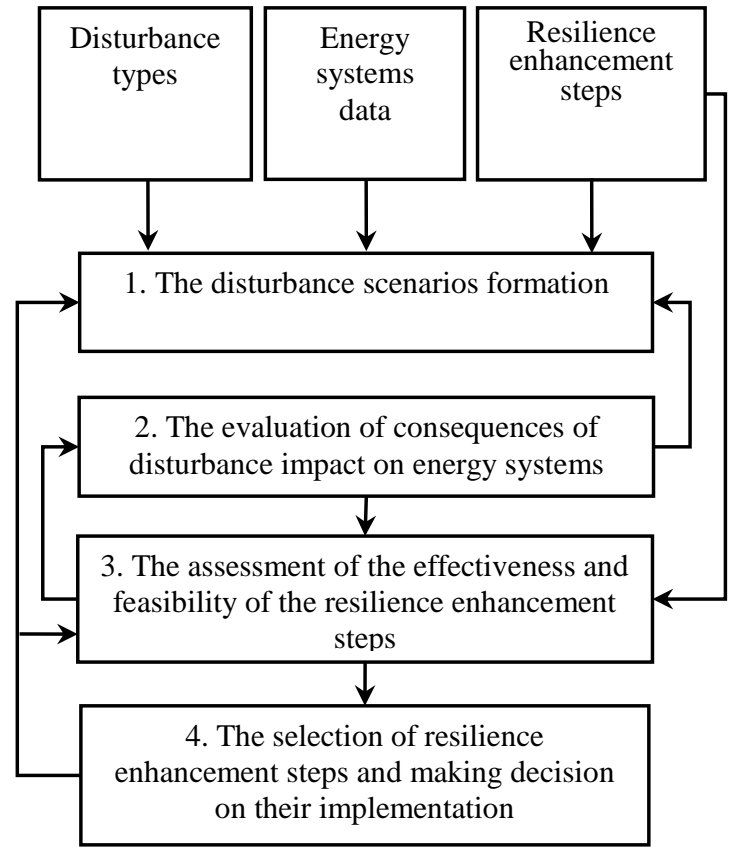

Figure 1. IIES resilience research scheme.

The third block assesses the effectiveness and feasibility of the resilience enhancement steps. One of the main effects of improving the IIES resilience is the reduction of economic and social damage because of the energy shortage.

The forth block includes the selection of resilience enhancement steps and the making decision on their implementation. The selected steps should be invariant to several subsets of conditions and types of disturbances.

According to [12] there are some challenges regarding IIES resilience. The first issue is the great uncertainty linked with penetration of distributed energy resources into the power generation mix. The second problem is the development of integrated approaches for system resilience enhancement [13- 
15]. Here the high performance computing [16-18] will help to solve combinatorial problems of resilience enhancement in a reasonable time.

\section{Instrumental platform}

The instrumental platform allows us to go to the automated process of design of IIES and implement the modeling of IIES. The basis of the developed instrumental platform is the concept of model-driven development (Model-Driven Engineering, MDE). The instrumental platform is developed as a result of adaptation to the problems being solved and the use of modern metaprogramming and ontology technologies. The MDE conception represents a set of methodological approaches to the automated design of complex software systems based on the preliminarily developed models. Within the framework of the proposed approach, MDE is adapted to the features of the subject area of computer simulation of IIESs. The automated construction of a software system is based on the computer model of the IIESs, computing subsystem model, and ontologies.

The attraction of modern metaprogramming technologies provides the possibility of automatically formation a software system. Another advantage of modern metaprogramming technologies is the possibility of flexible adjustment to the particularities of development and the composition of the IIES equipment, the resilience problem. The authors use reflective programming, which is one of the metaprogramming types. It represents an extension of the object-oriented programming paradigm. Reflective programming makes it possible to perform operations that cannot be performed within the object-oriented programming. Some of the most important operations are:

- Examination of classes during the program run, determination of their attributes, methods and constructors;

- Development of new copies of objects based on the class name, with the use of constructors;

- Assignment and calculation of values of attributes by their names; call of methods by their name and description of arguments;

- Flexible work with arrays and containers (collections).

Reflective programming allows inspecting the software system structure, and dynamically change the set of software components during its operation.

A feature of modeling problems of IIESs is that the implementation of software, which is designed to solve applied problems, is the final stage of developing methods, mathematical models, methods and algorithms. The use of this software leads to an accumulation of experience which enables:

- To develop more accurate mathematical models, refine reference information,

- Increase the speed of algorithms, improve the convergence of methods,

- Get the original solution of a practical problem.

As a rule, the accumulated experience is recorded by making changes in the software. It increases the quality of software and compliance with real engineering systems. The described approach to software development leads to the fact that software becomes the only means of formalizing and storing all the accumulated experience. As a result, this experience is not available for the study and use of a wide range of specialists. The authors proposed to carry out a formal description of the knowledge of the subject area in the form of ontologies, which will make it possible to reuse them in the automation of the construction of applied software.

The ontologies are used to solve the following problems:

- Automated construction of a program system,

- Create the user interface content,

- Application of the software system for solving applied problems.

Methods of building applied ontologies will be applied to automate the construction of software components and to control the process of mathematical modeling of IIESs. The ontologies contain knowledge about IIESs, applied problems and software that is designed to solve them. XML is used as a means of describing ontologies. SVG (Scalable Vector Graphics - scalable vector graphics) is used to describe graphic models of objects of energy systems. MathML (Mathematical Markup Language mathematical markup language) is used to describe mathematical expressions. 
When developing methodological approaches and software implementation, the authors focus only on free software. Java is used as a basic programming language, the choice of which is due to the presence of the following advantages:

- Support for modern technologies of object-oriented, component and functional programming,

- Native support for metaprogramming technologies,

- A wide range of technologies and tools for organizing distributed and parallel computing.

The Java platform provides a unified universal technology to access JDBC databases (Java DataBase Connectivity). The project is supposed to use the OpenJDK (Open Java Development Kit), which is a free and open implementation of the Java SE platform.

The GNU Compiler Collection (GCC), a set of compilers for various programming languages developed as part of the GNU project, is supposed to be used to compile software modules in $\mathrm{C}, \mathrm{C}++$, and Fortran.

As the main software development tool for the Project, Eclipse is supposed to be used - a free integrated software development environment that supports a large number of programming languages (Java, C, C ++, Fortran, etc.) and developed by the Eclipse Foundation.

Firebird is used as a DBMS, which has multi-user access and portability between different operating systems. Firebird is based on InterBase 6.0 source code, which was released as Open Source by Borland in August 2000. To access databases from Java applications, it is supposed to use the official driver for the FireBird DBMS - JayBird.

\section{Energy system resilience research}

The approach for energy system resilience research proposed in [19] is built on the top of a modeling framework for interdependent technical infrastructures [20,21]. This framework enables representing dependencies of various types between infrastructures [22-24] and describing natural disasters, manmade catastrophes, technical failures, international crime and terrorism as either structural or functional disturbances. A main advantage of the modeling framework is that it gives a common platform for modeling different technical infrastructures and simulating their interactions [25].

As disturbances can affect either the structural or functional properties of the system then, at first step, each technical infrastructure is divided into two main parts:

- The structural part of the system with the interconnections of system components,

- The functional part that controls flow over the infrastructure network.

In the structural model, the system's physical components are represented as nodes and arcs. In the functional model, the system overall performance is evaluated taking into account both the topological and functional constraints. The functional model can range from simple topological models to advanced dynamical models.

The first step provides the same fundamental way for modeling interdependent technical infrastructures since each system is initially modeled as the single infrastructure considering its direct dependencies to other infrastructures [25]. On the second step, these individual models are coupled together to form a "system-of-systems" [26-28] to study the effects of interdependencies between the systems.

Vulnerability analysis plays the central role in support to decision making for proper energy system protection and for guaranteeing energy system resilience [13]. According to [20] the concept of vulnerability has two closely related interpretations in the research literature. In the first interpretation, vulnerability is seen as a global system property. That property expresses the extent of negative effects caused by the occurrence of a specific disturbance. In the second interpretation, vulnerability is used to describe a system component if the failure of that component causes large negative consequences to the system.

\section{Distributed computing environment}

The integrated approach provides [19] the quantification of the energy system resilience, the identification of their critical elements, and allows the evaluation and comparison of the effectiveness of different protection and recovery strategies. It requires that the vast number of scenarios must be 
analyzed and evaluated to cover as many disturbances as possible [25]. To overcome this challenge a heterogeneous distributed computing environment was developed [19].

Depending on the dimensions of the used models, this problem can be solved using different computing systems (personal computer, server, cluster, grid, or cloud) for a relatively acceptable time (hours, days), the duration of which is determined by the characteristics of the used computing resources. Integration of the listed systems into a single environment provides both the flexibility in selecting the necessary configuration of the computational infrastructure and speed in implementing experiments of various scales.

Figure 2 shows the integration scheme of computational systems into the single problem-oriented environment for solving problems of studying the energy system resilience. The integration is carried out with the help of tools to develop distributed applied software packages like Orlando Tools.

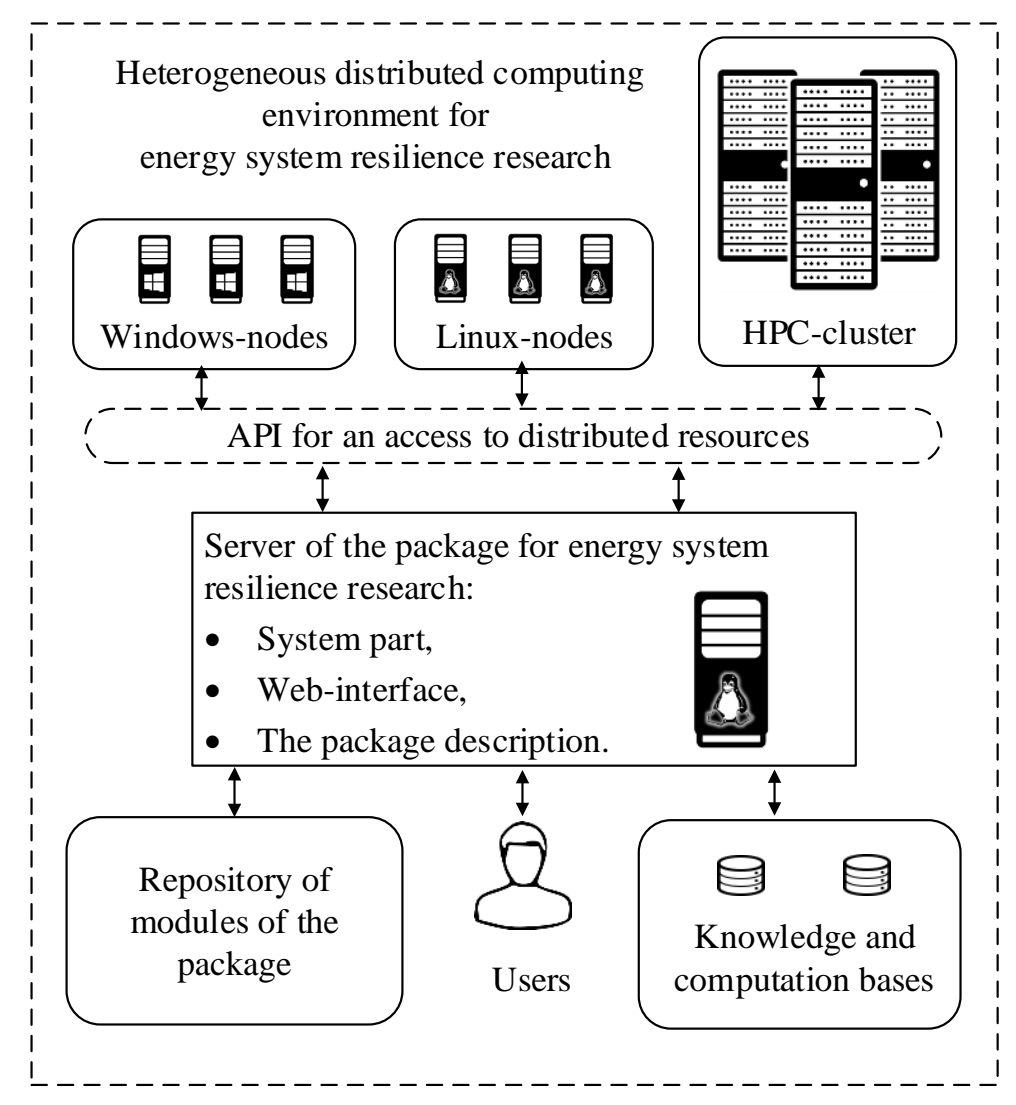

Figure 2. Heterogeneous distributed computing environment.

Orlando Tools provides the declarative specification of algorithmic knowledge, data about software and hardware of environment nodes, and information about administrative policies in them. Algorithmic knowledge includes computational knowledge about modules for solving problems in the subject domains of packages, schematic knowledge about the modular structure of models and algorithms, production knowledge to support making decision in selecting optimal algorithms for solving the problem. Orlando Tools uses XML notation as the main input language.

To simplify the description of package computational model for the subject domain experts, a highlevel Computational Model Description Language (CMDL) was developed. A tool to convert CMDL description to XML notation was implemented with the PEG.js [29]. The converter allows not to change the Orlando Tools for developing packages but to enhance them with new features. The CMDL syntax corresponds to the concepts of a subject domain: package, parameter, operation, module, and problem formulation. 
To increase development efficiency, the possibilities of continuous integration of application packages available in Orlando Tools were used. These means allowed to organize a unified chain in testing the entire distributed application, thereby reducing the involvement of developers in this timeconsuming process, and allowing them to spend more time developing the capabilities of the distributed application on the whole.

\section{Conclusions}

The current state of the IIES resilience study can be characterized by the following disadvantages:

- Focusing on the individual energy systems and neglecting their interdependencies [30],

- Cyber-physical nature of IIES is not fully considered [31].

Thus, an integrated approach is needed to take into account the peculiarities of the modeling IIES $[32,33]$ at various levels of the territorial and technological hierarchy that allows studying their interaction under large disturbances impact on the single platform [34]. Taking into account the structural and dynamic complexity of IIES, the characterization of the structural and functional disturbances, the evaluation of their consequences and probabilities there is not one single modeling approach that captures all aspects of IIES behavior. The integrated approach for energy system resilience research unites a number of existing methods and new analysis approaches capable of viewing the IIES complexity problem from different points of view, under the large uncertainties exist in the description of the failure behaviour of the elements of IIES, of their interconnections and interactions [13-15].

The integration of heterogeneous distributed computing environment and IIES design instrumental platform allows solving new problems of the comprehensive resilience analysis of IIES based on a single system modeling approach taking into account higher order (inter)dependencies between them.

\section{Acknowledgments}

The research was carried out under State Assignment, projects no. III.17.4.1 (reg. no. AAAA-A17117030310432-9) and no. III.17.5.1 (reg. no. AAAA-A17-117030310451-0) of the Fundamental Research of Siberian Branch of the Russian Academy of Sciences. In addition, it was supported in part by the Russian Foundation for Basic Research, projects no. project №18-51-06001 (reg. no. AAAAA18-118050490009-5) and no. 19-07-00097-a (reg. no. AAAA-A19-119062590002-7). The development of a technology for integrating Grid and cloud computing was supported by the Presidium RAS, program no. 2, project "Methods and tools for solving hard-search problems with supercomputers" (reg. no. AAAA-A18-118031590005-5).

\section{References}

[1] Voropai N I, Stennikov V A and Barakhtenko E A 2018 Methodological principles of constructing the integrated energy supply systems and their technological architecture Journal of Physics: Conference Series 1111(1) p 012001

[2] Hosseini S, Barker K and Ramirez-Marquez J E 2016 A review of definitions and measures of system resilience Reliability Engineering \& System Safety 145 pp 47-61

[3] Sansavini G 2017 Engineering resilience in critical infrastructures Resilience and Risk (Dordrecht: Springer) pp 189-203

[4] Sheffi Y 2005 The resilience enterprise: overcoming vulnerability for competitive enterprise (Cambridge: MIT Press)

[5] Pregenzer A 2011 Systems resilience: a new analytical framework for nuclear nonproliferation (Albuquerque, NM: Sandia National Laboratories)

[6] National Infrastructure Advisory Council (US) 2009 Critical infrastructure resilience: Final report and recommendations

[7] Carlson J L, Haffenden R A, Bassett G W, Buehring W A, Collins M J, Folga S M, Petit F D, Phillips J A, Verner D R and Whitfield R G 2012 Resilience: Theory and Application (Argonne National Laboratory) 10.2172/1044521

[8] Sharifi A and Yamagata Y 2016 Principles and criteria for assessing urban energy resilience: A literature review Renewable and Sustainable Energy Reviews 60 pp 1654-1677

[9] Lin Y, Bie Z and Qiu A 2018 A review of key strategies in realizing power system resilience 
Global Energy Interconnection 1(1) pp 70-78

[10] Roege P E, Collier Z A, Mancillas J, McDonagh J A and Linkov I 2014 Metrics for energy resilience Energy Policy $\mathbf{7 2}$ pp 249-256

[11] Barakhtenko E, Sokolov D, Edelev A and Gorsky S 2019 Vulnerability Analysis of Intelligent Integrated Energy Systems Proceedings 7th Scientific Conference on Information Technologies for Intelligent Decision Making Support (Atlantis Press) pp 74-76.

[12] Huang G, Wang J, Chen C, Guo C and Zhu B 2017 System resilience enhancement: Smart grid and beyond Front Eng Manag 4(3) pp 271- 282

[13] Zio E 2016 Challenges in the vulnerability and risk analysis of critical infrastructures Reliability Engineering \& System Safety 152 pp 137-150

[14] Zio E 2016 Critical infrastructures vulnerability and risk analysis European Journal for Security Research 1(2) pp 97-114

[15] Zio E 2016 Reliability analysis of systems of systems IEEE Reliability Magazine pp 1-6.

[16] Sharma T, Glynna J, Panosd E, Deanea P, Gargiuloe M, Rogana F and Gallachoir B O 2019 High performance computing for energy system optimization models: Enhancing the energy policy tool kit Energy Policy 128 pp 66-74

[17] Wang Y, Wang S and Wu L 2017 Distributed optimization approaches for emerging power systems operation: A review Electric Power Systems Research 144 pp 127-135

[18] Khaitan S K 2016 A survey of high-performance computing approaches in power systems 2016 IEEE Power and Energy Society General Meeting (IEEE) pp 1-5

[19] Edelev A, Sidorov I and Feoktistov A 2018 Heterogeneous Distributed Computing Environment for Vulnerability Analysis of Energy Critical Infrastructures Vth International workshop" Critical infrastructures: Contingency management, Intelligent, Agent-based, Cloud computing and Cyber security"(IWCI 2018) (Atlantis Press)

[20] Johansson J and Hassel H 2010 An approach for modeling interdependent infrastructures in the context of vulnerability analysis Reliability Engineering \& System Safety, 95(12) pp 13351344

[21] Svegrup L, Johansson J and Hassel H 2019 Integration of Critical Infrastructure and Societal Consequence Models: Impact on Swedish Power System Mitigation Decisions Risk Analysis

[22] Dudenhoeffer D D, Permann M R, Manis M 2006 CIMS: a framework for infrastructure interdependency modeling and analysis Proceedings of the 2006 winter simulation conference pp 478-485

[23] Zhang P and Peeta S 2011 A generalized modeling framework to analyze interdependencies among infrastructure systems Transp Res Part B: Methodological 45(3) pp 553-579

[24] Rinaldi S M, Peerenboom J P and Kelly T K 2001 Identifying, understanding, and analyzing critical infrastructure interdependencies IEEE Control Systems 21(6) pp 11-25

[25] Johansson J and Hassel H 2012 Modeling, simulation and vulnerability analysis of interdependent technical infrastructures Risk and Interdependencies in Critical Infrastructures: A Guideline for Analysis.ed P Hokstad, I B Utne and J Vatn (London: Springer-Verlag) pp 49-66

[26] Eusgeld I, Nan C and Dietz S 2011 "System-of-systems" approach for interdependent critical infrastructures Reliability Engineering \& System Safety 96(6) pp 679-686

[27] Mittal S, Ruth M, Pratt A, Lunacek M, Krishnamurthy D and Jones W 2015 A system-of-systems approach for integrated energy systems modeling and simulation Proceedings of the Conference on Summer Computer Simulation (Society for Computer Simulation International) pp 1-10

[28] Thacker S, Pant R and Hall J W 2017 System-of-systems formulation and disruption analysis for multi-scale critical national infrastructures Reliability Engineering \& System Safety 167 pp 3041

[29] Parser Generator for JavaScript. [Online]. Available: https://pegjs.org.

[30] Lin Y and Bie Z 2016 Study on the Resilience of the Integrated Energy System Energy Procedia 103 pp 171-176 
[31] Mihalache S F, Pricop E and Fattahi J 2019 Resilience Enhancement of Cyber-Physical Systems: A Review Power Systems ed N Mahdavi Tabatabaei, S Najafi Ravadanegh, N Bizon (Cham: Springer)

[32] Stennikov V, Barakhtenko E and Sokolov D 2018 Automation of the Integrated Graphical Environment Construction Advances in Intelligent Systems Research 158 pp 152-157

[33] Stennikov V, Barakhtenko E and Sokolov D 2018 The Use of Ontologies in the Integrated Graphical Environment Advances in Intelligent Systems Research 158 pp 158-163

[34] Edelev A V, Senderov S M, Beresneva N M, Sidorov I A and Feoktistov A G 2018 Distributed computing environment for an analysis of the vulnerability of critical infrastructures in energy sector Systems of Control, Communication and Security 3 pp 197-231 Available at: http://sccs.intelgr.com/archive/2018-03/10-Edelev.pdf (accessed 10 September 2018) (in Russian). 